\title{
Evaluation of resistance to pyrethroid and organophosphate adulticides and $k d r$ genotyping in Aedes aegypti populations from Roraima, the Northernmost Brazilian State
}

\author{
Ramão Luciano Nogueira Hayd \\ Universidade Federal de Roraima \\ Luana Carrara \\ Fundacao Oswaldo Cruz \\ Joel de Melo Lima \\ Secretaria de Estado de Saúde de Roraima \\ Nathalia Coelho Vargas de Almeida \\ Secretaria de Estado da Saude de Roraima \\ José Bento Pereira Lima
}

Fundacao Oswaldo Cruz

Ademir Jesus Martins ( $\sim$ ademirji@ioc.fiocruz.br)

Fundacao Oswaldo Cruz https://orcid.org/0000-0001-5739-1215

\section{Research}

Keywords: Vector Control, Insecticide Resistance Monitoring, Rorainópolis, Pacaraima, Bonfim, Boa vista, Deltamethrin, Malathion

Posted Date: April 1st, 2020

DOI: https://doi.org/10.21203/rs.2.21242/v2

License: (c) (i) This work is licensed under a Creative Commons Attribution 4.0 International License. Read Full License

Version of Record: A version of this preprint was published at Parasites \& Vectors on May 20th, 2020. See the published version at https://doi.org/10.1186/s13071-020-04127-w. 


\section{Abstract}

Background. Roraima, the northernmost State in Brazil, borders Venezuela and Guyana. Although mostly covered by the tropical forests, the urban centers of this State are highly infested with Ae. aegypti and endemic for dengue, Zika and chikungunya. Herein we present the levels of Ae. aegypti infestation and number of arboviruses cases between 2015 and 2018 in the studied localities. We accessed the insecticide resistance status of Ae. aegypti populations from the capital Boa Vista, two cities on international borders (Pacaraima and Bonfim) and Rorainópolis bordering Amazonas State, in order to evaluate the chemical control efficacy in these localities.

Methods. Tests with World Health Organization (WHO)-like tubes impregnated with the pyrethroid deltamethrin $(0.05$ and $0.12 \%)$ and the organophosphate malathion $(0.7 \%)$ were conducted with $A e$. aegypti from Boa Vista, Pacaraima, Bonfim and Rorainópolis, collected in 2016 and 2018. Genotyping of $k d r$ mutations, related to resistance to pyrethroids, was performed for the SNP variations in the sites 1016 and 1534 of the voltage gated sodium channel gene $\left(\mathrm{Na}_{\mathrm{V}}\right)$ with a TaqMan qPCR approach.

Results. Ae. albopictus was absent in our collections, and therefore only Ae. aegypti was tested. All Ae. aegypti populations were susceptible to $0.7 \%$ malathion in 2016 , however mortality dropped to under $90 \%$ in Bonfim and Pacaraima populations in 2018. All populations were resistant to $0.05 \%$ deltamethrin in both years. The time that $50 \%$ of females suffered knockdown ( $\left.K d T_{50}\right)$ under exposition to $0.05 \%$ deltamethrin was 3.3-5.9 fold longer in mosquitoes from the populations compared to the susceptible strain Rockefeller. Only Pacaraima population (2018) remained resistant to $0.12 \%$ deltamethrin. The $k d r$ genotyping revealed the absence of the wild-type $\mathrm{Na}_{\mathrm{V}} \mathrm{S}$ haplotype (1016Val +1534Phe) in the populations from Roraima, signifying that all tested insects had a genetic background for pyrethroid resistance. The double $k d r \mathrm{Na}_{\vee} \mathrm{R} 2$ haplotype (1016lle $\left.+15434 \mathrm{Cys}\right)$ was present in higher frequencies in all populations except Rorainópolis, where this haplotype seems to have arrived recently.

Conclusions. These results are important for the knowledge about insecticide resistance status of $A e$. aegypti populations from Roraima and will help to improve vector control strategies that may be applied to diverse localities under similar geographical and urban conditions.

\section{Introduction}

The globalization and rapid adaptation of Aedes aegypti to urban conditions are favoring its dispersion around the globe, posing a serious threat to human public health since this mosquito is a vector of many arboviruses such as dengue, Zika, chikungunya and urban yellow fever, among others [1]. Roraima is a State in the extreme North of Brazil bordering Venezuela and Guyana and is therefore an important Brazilian doorway for the entrance or emergent and re-emergent arbovirus, given the circulation of wildlife, people and goods between the borders. In addition, population genetic studies have suggested that the re-infestation of Ae. aegypti in Brazil likely occurred from Venezuela during the 1970's [2], evidencing the needs of a constant entomological surveillance in Roraima State. 
In 2010, the re-emergence of DENV-4 in Brazil, with a hypothesized origin in Venezuela, was first recorded in Boa Vista, the capital of Roraima, rapidly spreading to other States in the country: Amazonas and Pará (North), Bahia, Pernambuco and Piauí (Northeast) as well as Rio de Janeiro and São Paulo (Southeast) [3]. Attempting to block the DENV-4 circulation, efforts targeted the elimination of larval breeding sites and the intensification of chemical control with employment of the insect growth regulator diflubenzuron and the pyrethroid adulticide deltamethrin. However, this strategy did not significantly reduce Ae. aegypti infestation and rapidly increased the levels of insecticide resistance to the pyrethroid [4].

Insecticide resistance (IR) is a threat to the control of arboviruses at a global scale [5]. In Brazil, a nationwide IR monitoring program coordinated by the Ministry of Health has been screening the status of susceptibility to recommended chemicals in Ae. aegypti populations of strategic localities since 1999 [6]. In previous surveys, pyrethoid resistance was detected in Ae. aegypti from Boa Vista and Pacaraima [7, 8], probably due to selection of $k d r$ mutations. These mutations are single nucleotide polymorphisms (SNPs) in the voltage gated sodium channel gene $\left(\mathrm{Na}_{V}\right)$, which encodes a neuronal transmembrane protein that is the target of pyrethroids and DDT. In Ae. aegypti populations from Brazil, there are registers of at least four principal SNPs in the $\mathrm{Na}_{V}$ gene: V410L, I1011M, V1016I and F1534C $[8,9,10]$, whereas the latter two were more related to resistance to the pyrethroid knockdown effect $(k d r)$ [10]. All Ae. aegypti samples evaluated from Boa Vista and Pacaraima in 2011 harbored $k d r$ mutations, in either $\mathrm{Na}_{\mathrm{V}} \mathrm{R} 1$ (1016Val + 1534Cys $\left.{ }^{k d \eta}\right)$ or NavR2 (1016lle ${ }^{k d r}+1534$ Cys $\left.^{k d \eta}\right)$ haplotypes [8]. In addition to the presence of $k d r$ mutations, alterations in the activity of enzymes related to metabolic resistance, such as Glutathione-S tranferases (GSTs) and Esterases, were also observed in these populations [7]. Given resistance to pyrethroids detected in Ae. aegypti populations throughout the country, the $\mathrm{MoH}$ has replaced these compounds with the organophosphate malathion [7], which in Roraima State was introduced in 2015 by governmental campaigns. Pyrethroids however habitually continued being applied through household sprays vastly available in market stores and by governmental programs against malaria vectors in endemic regions.

Herein, we evaluate the profile of resistance to pyrethroid and organophosphate adulticides in Ae. aegypti populations of four important cities of Roraima State, Brazil, in 2016 and 2018, extending the analyses to localities not previously assessed. Kdrhaplotype frequency was also investigated.

\section{Methods}

Collections and laboratory rearing conditions. Roraima State has a territory of $224,273.83 \mathrm{Km}^{2}$ and is the least populous Brazilian State with only $0.29 \%$ of the total population in the country $(605,761$ inhabitants 
as stipulated by the Brazilian Institute of Geography and Statistics) [11]. Roraima has limits with the Amazon in the south and borders Venezuela and Guyana (Figure 1). The capital, Boa Vista, is $741.1 \mathrm{Km}$ from Manaus (the capital of Amazonas State) by BR-174 road. This same highway connects Boa Vista to Pacaraima on the border of Venezuela, 2,013.5 Km to the North.

Collections of eggs were performed in four of the most important cities of Roraima State, including the capital, Boa Vista $\left(02^{\circ} 49^{\prime} 11^{\prime \prime} \mathrm{N}, 60^{\circ} 40^{\prime} 24^{\prime \prime} \mathrm{W}\right)$, Bonfim $\left(02^{\circ} 45^{\prime} 22.25^{\prime \prime} \mathrm{N}, 60^{\circ} 7^{\prime} 6.53^{\prime \prime} \mathrm{O}\right)$ in the Northeast on the border with Guyana, Pacaraima $\left(04^{\circ} 25^{\prime} 01^{\prime \prime} \mathrm{N}, 61^{\circ} 08^{\prime} 27^{\prime \prime} \mathrm{O}\right)$ in the North on the border with Venezuela and Rorainópolis in the South on the Amazonas State limits $\left(00^{\circ} 56^{\prime} 22.62^{\prime \prime} \mathrm{N}, 60^{\circ} 26^{\prime} 21.91 " 0\right)$. Ovitraps consisted of $800 \mathrm{~mL}$ black plastic cups, containing $300 \mathrm{~mL}$ of a $0.04 \%$ yeast extract solution to attract gravid females which would lay their eggs in a wood paddle immersed in this solution for 5-7 days. The total of 150 traps were installed in Boa Vista and 50 in each of the other cities, following standard recommendations [12]. Paddles were shipped to the laboratory Núcleo de Pesquisa Observatório da Saúde at the Federal University of Roraima State (UFRR), eggs stimulated to hatch and larvae reared under laboratory conditions as described elsewhere [12]. Adults were screened for species identification and maintained in cardboard cylindrical cages $(17 \times 18 \mathrm{~cm})$ with a $10 \%$ sucrose solution w/v offered ad libitum. Anesthetized rats (Rattus norvegicus wistar) were offered to blood feed females in order to produce eggs of an F1 generation, following procedures as recommended in the license \# 12/2015 approved by the ethical committee on animal use of UFRR (CEUA-UFRR). The reference lineage Rockefeller mosquitoes maintained at Laficave/Fiocruz since 1999 [12] were raised in parallel and adopted in all assays as a control of insecticide susceptibility and vigor under test conditions.

$\underline{\text { Insecticide resistance related assays. }}$.

Bioassays. Bioassays were performed with $\mathrm{F} 1$ generation Ae. aegypti females $3-5$ days post-adult emergence with WHO tube tests [13]. We employed the pyrethroid deltamethrin technical grade (Sigma Aldrich, St. Louis, USA) dissolved in acetone to a $1 \%$ stock solution and then diluted it to the concentrations of 0.05 and $0.12 \%$ in silicone oil (Dow Corning, Midland, USA) as the solvent carrier. Malathion (Sangrose Agroquímica, Curitiba, Brazil) emulsion concentrate was diluted in the silicone oil to the concentration of $0.7 \%$ and subsequently applied over a $12 \times 15 \mathrm{~cm}$ filter paper (Whatman Grade 1 ) as previously described [14]. Mosquitos were exposed for 1 hour in the tube chamber with insecticide and then gently transferred to the resting chamber until mortality was registered $24 \mathrm{hs}$ later. In addition, knockdown rate was evaluated at every 5 minutes (or 2 minutes for Rockefeller) during the exposition to deltamethrin $0.05 \%$. A probit analysis [15] was incorporated in order to calculate the time when $50 \%$ of the females were knocked down $\left(K d T_{50}\right)$. The knockdown resistance ratios (RR $\left.K d T_{50}\right)$ of the populations were obtained by the quotient between the $K d T_{50}$ of the populations with Rockefeller, the control strain. 
Kdrgenotyping.

DNA was extracted from male mosquitoes and genotyped for the single nucleotide polymorphisms (SNPs) at the $1016\left(\mathrm{Val}^{+}\right.$or $l \mathrm{e}^{k d}$ ) and 1534 ( $\mathrm{Phe}^{+}$or Cys ${ }^{k d}$ ) sites of the voltage gated sodium channel gene $\left(\mathrm{Na}_{V}\right)$ using exactly the same procedures and reagents as described [16]. As these sites are linked in the same gene, both SNPs were considered in order to define the genotype frequencies for a single locus [8]. The haplotypes usually found in Ae. aegypti populations from Brazil are the wild-type $\mathrm{Na}_{\mathrm{V}} \mathrm{S}$ (1016Val + 1534Phe) and the kdrs Nav R1 (1016Val + 1534Cys) and $\mathrm{Na}_{\mathrm{V}} \mathrm{R} 2$ (1016lle + 1534Cys), whereas the possible genotypes are SS, SR1, SR2, R1R1, R1R2 and R2R2. As kdrmutations act as a recessive trait, insects resistant to pyrethroids based on this target site mechanism present one of the genotypes R1R1, R1R2 or R2R2. The NavR2 haplotype generates higher levels of resistance [14].

\section{Results}

Bioassays. We evaluated the susceptibility status of Ae. aegypti from four Roraima municipalities to the pyrethroid deltamethrin (0.05 and $0.12 \%)$ and organophosphate malathion (0.7\%) adulticides, in 2016 and 2018.

In 2016, populations exhibited some survival to malathion $0.7 \%$, however with mortality rates greater than $90 \%$, except that from Rorainópolis, attaining a mortality rate of $100 \%$ (Figure 2). There was a reduction in the mortality levels in 2018 in all localities, ranging from $84.8 \%$ in Pacaraima to $94.2 \%$ in Rorainópolis (Table 1). Therefore, Pacaraima and Boa Vista were classified as resistant to malathion in 2018.

\section{Table 1. Bioassays with the organophosphate malathion (0.7\%) in Aedes aegypti populations from Roraima State, Brazil (2016 and 2018).}

\begin{tabular}{lrrrr}
\hline Populations & \multicolumn{3}{c}{2016} & \multicolumn{2}{c}{2018} \\
& total N & mean mortality $\% \pm \mathrm{SD}$ & total Na & mean mortality \% \pm SD \\
& & & & \\
\hline Boa Vista & 175 & $97.8 \pm 2.48$ & 197 & $100 \pm 0$ \\
\hline Bonfim & 171 & $97.1 \pm 4.97$ & 181 & $85.6 \pm 16.77$ \\
\hline Rorainópolis & 185 & $95.9 \pm 2.37$ & 184 & $94.2 \pm 7.51$ \\
Pacaraima & 188 & $93.6 \pm 8.37$ & 178 & $84.8 \pm 25.01$
\end{tabular}


a Total number of insects used/ population

With regard to the pyrethroid, all populations were considered resistant both in 2016 and 2018 when evaluated with $0.05 \%$ deltamethrin. From 2016 to 2018 there was an increase in the mortality in Boa Vista (64.0 to 86.6\%) and Bonfim (31.2 to 78.5\%) with a decrease in Rorainópolis (89.9 to 74.3\%) and Pacaraima (78.3 to 61.7\%). A higher dosage of deltamethrin $(0.12 \%)$ was tested in parallel, and as expected, this higher concentration increased the mortality in all cases, however not reaching $100 \%$ of mortality in any population (Figure 3-A). We also evaluated the knockdown rate during the time of exposition to deltamethrin $0.05 \%$ for $1 \mathrm{~h}$ (Additional file Figure S1). According to a probit analysis, $50 \%$ of the Rockefeller females were knocked down $\left(K d \Gamma_{50}\right)$ by 11.9 minutes, compared to 29.6 and 69.9 minutes in Boa Vista (2018) and Bonfim (2016) populations, respectively (Table 2). Nevertheless, the $K d \Gamma_{50}$ values of all populations were similar if we consider the IC95\% interval, except Bonfim in 2016 which presented a higher $K d \Gamma_{50}$ (Figure 3-B). The knockdown-resistance ratio $\left(K d \Gamma-R_{50}\right)$ of the populations ranged from 2.5 to 5.9 (Table 2).

Table 2. Time of knockdown to the pyrethroid deltamethrin (0.05\%) in Aedes aegypti populations from Roraima State, Brazil (2016 and 2018).

\begin{tabular}{|c|c|c|c|c|c|c|c|c|}
\hline \multirow[t]{2}{*}{ Populations } & \multicolumn{4}{|l|}{2016} & \multicolumn{4}{|l|}{2018} \\
\hline & total $\mathrm{N}^{\mathrm{a}}$ & $K d \mathrm{~T}_{50} \min$ & (IC95\%) & $K d \mathrm{~T}-\mathrm{RR}_{50}$ & total $\mathrm{N}^{\mathrm{a}}$ & $K d \mathrm{~T}_{50} \min$ & (IC95\%) & $K d \mathrm{~T}-\mathrm{RR}_{50}$ \\
\hline Boa Vista & 320 & 40.6 & $(34.7-47.5)$ & 3.4 & 178 & 29.6 & $(24.9-35.3)$ & 2.5 \\
\hline Bonfim & 146 & 69.9 & $(52.9-92.4)$ & 5.9 & 172 & 34.4 & $(28.8-41.2)$ & 2.9 \\
\hline Rorainópolis & 187 & 40.6 & $(34.6-47.8)$ & 3.4 & 177 & 42.7 & (38.1-47.9) & 3.6 \\
\hline Pacaraima & 182 & 39.8 & (35.4-44.7) & 3.3 & 193 & 42.1 & $(35.1-50.6)$ & 3.5 \\
\hline Rockefeller & 182 & 11.9 & $(8.6-16.5)$ & & & & & \\
\hline
\end{tabular}

a Total number of insects used/ population

$\underline{K d r}$ genotyping. We successfully genotyped 275 Ae. aegypti mosquitoes from Roraima State for both 1016 (Val+ or Ile ${ }^{k d r}$ ) and 1534 (Phe $^{+}$or Cys ${ }^{k d r}$ ) sites. All tested insects demonstrated a $k d r$ mutation in at least one site, presenting genotypes: homozygotes for the $1534 k d r$ (R1R1) and the double $k d r 1016+1534$ (R2R2) in addition to the heterozygote 
(R1R2). Therefore, there was no evidence of the wild-type haplotype S $(1016 \mathrm{Val}+1534 \mathrm{Phe})$ in Ae. aegypti populations from Roraima (Figure 4-A).

The double $k d r$ haplotype R2 (1016 Ile + 1534 Cys) was in higher frequency than R1 (1016 Val + 1534 Cys) in Boa Vista (2018), Bonfim (2016 and 2018) and Pacaraima (2016 and 2018) (Table 3). In Rorainópolis, R2 was under low frequencies (8 and 11\%, respectively in 2016 and 2018), only appearing in heterozygotes R1R2 (Figure 4-B). In all cases, the genotypic frequencies did not deviate from the Hardy-Weinberg Equilibrium assumption (Table 3).

When comparing the genotypic frequencies between the 2016 and 2018 collections, the R2R2 genotype decreased in Boa Vista (25 to 18.6\%), Bonfim (45 to 36.7\%) and particularly in Pacaraima (45.5 to 20.7\%). Indeed as aforementioned, the levels of mortality to deltamethrin 0.05\% increased in both Boa Vista and Bonfim, however decreasing in Pacaraima (Figure 3-B). We also genotyped some mosquitoes dead (susceptible) and alive (resistant) $24 \mathrm{hs}$ after $1 \mathrm{~h}$ of exposition to deltamethrin $0.05 \%$ from Boa Vista and Bonfim. Although the number of samples was small (41 alive and 14 dead), in the resistant group the R1R1 genotype was absent while R2R2 was more representative (Figure 4-C).

\section{Table 3. Frequencies of $k d r$ genotypes in Aedes aegypti populations from Roraima State, Brazil (2016 and 2018).}

\begin{tabular}{lllllllllll}
\hline & & \multicolumn{8}{c}{ Genotypic frequency } & \multicolumn{3}{c}{ Allelic frequency } & \multicolumn{2}{l}{ HWE test $^{\mathrm{b}}$} \\
\hline Locality & year & $\mathbf{N}^{\mathrm{a}}$ & $\mathbf{R 1 R 1}$ & $\mathbf{R 1 R 2}$ & $\mathbf{R 2 R 2}$ & $\mathbf{S}$ & $\mathbf{R 1}$ & $\mathbf{R 2}$ & $\mathrm{c} 2$ & $\mathbf{P}$ \\
Boa Vista & 2016 & 40 & 0.225 & 0.525 & 0.250 & 0 & 0.488 & 0.513 & 0.1 & 0.749 \\
\hline & 2018 & 43 & 0.279 & 0.535 & 0.186 & 0 & 0.547 & 0.453 & 0.3 & 0.604 \\
\hline Bonfim & 2016 & 40 & 0.150 & 0.400 & 0.450 & 0 & 0.350 & 0.650 & 0.6 & 0.445 \\
\hline \multirow{2}{*}{ Rorainópolis } & 2016 & 43 & 0.837 & 0.163 & 0 & 0 & 0.919 & 0.081 & 0.3 & 0.561 \\
\hline & 2018 & 28 & 0.786 & 0.214 & 0 & 0 & 0.893 & 0.107 & 0.4 & 0.525 \\
\hline Pacaraima & 2016 & 29 & 0.172 & 0.621 & 0.207 & 0 & 0.483 & 0.517 & 0.1 & 0.749 \\
\hline & 2018 & 29 & 0.138 & 0.655 & 0.207 & 0 & 0.466 & 0.534 & 0.3 & 0.604 \\
\hline
\end{tabular}

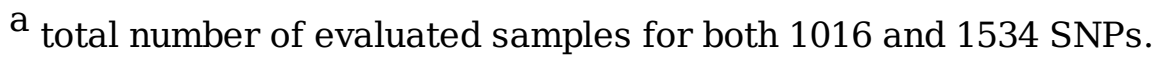




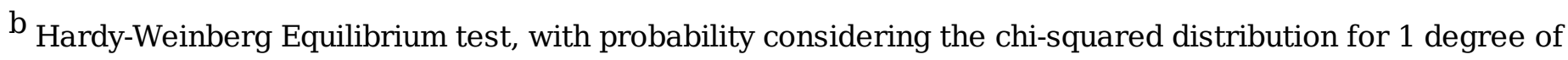
freedom.

\section{Discussion}

Herein we showed that Ae. aegypti populations from Roraima State, Brazil collected in 2016 and 2018 were resistant to the pyrethroid deltamethrin and under the process of becoming resistant to the organophosphate malathion. Remarkably, only Ae. aegypti was present in these collections, regardless of a recent register of $A$ e. albopictus in a rural area of Rorainopolis.

Pyrethroid resistance in Ae. aegypti from Roraima State had already been high in previous evaluations. The first registers were in 2007 and 2010 when the capital Boa Vista was detected as pyrethroid resistant $[4,17]$. In 2011, Pacaraima presented the second highest resistance ratio $\left(R R_{95}=60.3\right)$ in the whole country [7]. Herein we demonstrate that even two years after pyrethroid governmental application was substituted by malathion, pyrethroid resistance has been maintained in the four localities evaluated. It is noteworthy that a new diagnostic dose was established for deltamethrin $(0.03 \%)$ in WHO paper tests [13]. In this study we adopted a higher dosage $(0.05 \%)$ signifying that the rate of mortality would probably be even lower if tested with $0.03 \%$ deltamethrin papers.

The Brazilian MoH started replacing pyrethroids with the organophosphate malathion in ultra-low volume-based applications against Ae. aegypti in 2010 [7] although pyrethroids are still used in campaigns against other vectors such as anophelines, phlebotomines and tritomines. In Roraima State the first stock of malathion was received in December 2015. Hereing we declare that all four Ae. aegypti populations collected in 2016, although not $100 \%$ killed by malathion $0.7 \%$, had mortality above $90 \%$, then not classified as resistant. The rate of mortality decreased to under $90 \%$ in Bonfim and Pacaraima two years afterwards, the mosquitoes therefore being classified as resistant to malathion. The diagnostic dose for malathion indicated by WHO is a bit higher, $0.8 \%$ [13]. Had we used $0.8 \%$ instead of $0.7 \%$, the decrease in the mortality levels from 2016 to 2018 might have also been noticeable.

Different from that which occurs with pyrethroids, the active ingredient of household sprays and other governmental campaigns, the only probable source of organophosphate pressure was the governmental campaigns with malathion at that moment. The larvicide temephos has in theory not been applied in Roraima since 2013, when it was definitively substituted by IGRs [7]. The most recent data about temephos resistance in Roraima indicated resistance ratios $\left(\mathrm{RR}_{50}\right)$ of 2.0 in Boa Vista (2010) and 4.3 in Pacaraima (2011), which were not considered high levels of resistance $[4,17]$. Anyway, we cannot reject the possibility that mechanisms prior selected by temephos and pyrethroids are inducing cross resistance 
to malathion, as reported in some classical studies. For instance, a laboratory strain of Culex quinquefasciatus selected for temephos resistance in larvae developed cross resistance to several organophosphate adulticides, including malathion [18]. On the other hand, in Guadeloupe and Saint Martin Caribbean islands, Ae. aegypti populations developed high levels of resistance to temephos (8.9 to 33.1-fold) but low levels to malathion (1.7 to 4.4 -fold) [19]

Concerning the possible mechanisms selected for insecticide resistance, alterations in the activity of GST and Esterase enzymes were detected in Boa Vista (2007) and Pacaraima (2011). Reduced activity of the Acetylcholinesterase enzyme was also observed in Pacaraima (2011) [7]. Lineages of Ae. aegypti Brazilian populations that acquired resistance to malathion through selection pressure in the laboratory exhibited increased activity of GST, multi-function Oxidases (MFO P450) and Estereses, as determined by bichoemical analyses [20]. Over expression of genes related to metabolic resistance was detected in Ae. aegypti populations from the Caribbean, such as French Guyana and French West Indies islands [19, 21]. On the other hand, those high levels of resistance to pyrethroids in Roraima might be partially justified by the absence of the wild-type $\mathrm{Na}_{\mathrm{V}} \mathrm{S}$ haplotype, already reported in 2010 and 2011 in Boa Vista and Pacaraima [8]. The wild-type $\mathrm{Na}_{\mathrm{V}} \mathrm{S}$ haplotype is still missing with predominance of the double $k d r \mathrm{Na}_{\mathrm{V}} \mathrm{R} 2$ (1016lle + 1534Cys), except in Rorainópolis, where the Na $\mathrm{V}_{\mathrm{V}} 1$ (1016Val + 1534Cys) predominates. We corroborated that $\mathrm{Na}_{V} \mathrm{R} 2$ leads to higher levels of resistance to pyrethroids [14] once homozygote R2R2 insects were only present among the survivors in the bioassays with Boa Vista and Bonfim. The remaining high levels of resistance to pyrethroids even after the substitution by malathion in Roraima may be associated with the high prevalence of domestic use of insecticides, all composed of pyrethroids and easily acquired in local markets, as reported in other Brazilian states [16, 22]. In addition, we cannot neglect the migration of $A$ e. aegypti resistant populations from the neighboring countries and measures adopted against other vector borne diseases. On the border between Pacaraima (Brazil) and Santa Helena (Venezuela) there is an intense control of malaria where pyrethroids are employed against Anopheles even in the urban area, thus also submitting Ae. aegypti to this selection pressure. In Bonfim, it is common to find pyrethroid sprays acquired in Lethen City, on the Guyanese side of the border.

We speculate that the $k d r \mathrm{Na}_{\mathrm{V}} \mathrm{R} 2$ haplotype in Ae. aegypti populations in this region must have migrated mostly from Venezuela as it has been detected in Roraima State at least since 2010, when it either absent or at low frequencies in the neighboring States, Amazonas and Pará [8]. Interestingly, Rorainópolis is the only municipality in Roraima with Ae. albopictus colonization, however encountered in areas on the border with Amazonas State where the species has been recorded since 2015 (CGVS-SESAU-RR). Therefore, it seems that the transition between the biomes, Amazon Forest in the south and the savannah-like "Lavrado" in the north, has been limiting the dispersion of Ae. aegypti from Roraima downwards to Amazonas State. On the other hand, Ae. albopictus from Amazonas has avoided invasion of the capital Boa Vista in the Lavrado zone. 
Besides new promising tools of Ae. aegypti control currently being tested in Brazil, such as Wolbachia, RIDL (Release of Insects carrying a Dominant Lethal gene) and pyriproxyfen autodissemination stations $[23][24,25,26]$, insecticides continue to play an important role on a long term basis in order to rapidly decrease the densities of a target population and consequently, mitigate the cycle of the arboviruses. Therefore, it is of prime importance that a constant surveillance of insecticide resistance be inherent to the chemical control strategy so as to guide authorities about product efficacy.

\section{Additional File Legend}

Additional File - Figure S1. Curve of knockdown to deltamethrin $\mathbf{0 . 0 5 \%}$. Ae. aegypti populations from Boa Vista, Bonfim, Rorainópolis and Pacaraima, collected in 2016 and 2018, additional to the reference lineage Rockefeller. Red dotted line indicates the $K d T_{50}$.

\section{Abbreviations}

DDT: dichlorodiphenyltrichloroethane; $\mathrm{Na}_{v}$ : voltage-gated sodium channel gene; WHO: World Health Organization; MoH: Ministry of Health; LIRAa: quick survey of Aedes aegypti indexes; IGR: insect growth regulator; Kdr. knockdown resistance; RR: resistance ratio; KdT: time of knockdown; SNP: single nucleotide polymorphism.

\section{Declarations}

\section{Acknowledgements}

We thank Pedro Eduardo Lima Siqueira and Emilly Habert (UFRR) for their support with the bioassays and Heloisa Diniz, Instituto Oswaldo Cruz, for taking care of the figures in this publication. English reviewe and revision by Mitchell Raymond Lishon, native of Chicago, Illinois, U.S.A. - UCLA 1969.

\section{Declarations}

\section{Ethics approval and consent to participate}

Animals (Rattus norvegicus wistar) were used in this study in order to blood feed female mosquitos to produce laboratory colonies. Animals were maintained and anesthetized prior to blood feeding, accordingly to the procedures indicated by the ethical committee on animal use of Federal University of Roraima (CEUA-UFRR), approved license \# 12/2015. 


\section{Consent for publication}

Not applicable.

\section{Availability of data and materials}

All data generated or analyzed during this study are included in this published article [and its supplementary information files]. Any additional dataset are available upon request to the corresponding author.

\section{Competing interests}

The authors declare that they have no competing interests.

\section{Funding}

National Institutes of Health (NIH, Grant no. U01 Al115595), Instituto Nacional de Ciência e Tecnologia em Entomologia Molecular (INCT-EM, Grant no. 465678/2014-9), and Fundação de Amparo à Pesquisa do Estado do Rio de Janeiro (FAPERJ, Grant no. E-26/202.795/2019).

\section{Author contributions:}

Conceptualization: RLNH, AJM

Data curation: RLNH, LC

Formal Analysis: RLNH, AJM

Funding acquisition: RLNH, JBPL, AJM

Investigation: RLNH, AJM, JML, NCVA

Methodology: LC, JBPL, AJM

Project administration: AJM

Resources: JBPL, AJM

Supervision: AJM 
Validation: AJM

Visualization: all authors

Writing - original draft: RLNH, AJM

Writing - review \& editing: all authors

\section{All authors read and approved the final manuscript.}

\section{References}

1. Kraemer MUG, Reiner RC, Jr., Brady OJ, Messina JP, Gilbert M, Pigott DM, et al. Past and future spread of the arbovirus vectors Aedes aegypti and Aedes albopictus. Nat Microbiol. 2019;4 5:854-63.

2. Kotsakiozi P, Gloria-Soria A, Caccone A, Evans B, Schama R, Martins AJ, et al. Tracking the return of Aedes aegypti to Brazil, the major vector of the dengue, chikungunya and Zika viruses. PLoS Negl Trop Dis. 2017;11 7:e0005653.

3. de Souza RP, Rocco IM, Maeda AY, Spenassatto C, Bisordi I, Suzuki A, et al. Dengue virus type 4 phylogenetics in Brazil 2011: looking beyond the veil. PLoS Negl Trop Dis. 2011;5 12:e1439.

4. Maciel-de-Freitas R, Avendanho FC, Santos R, Sylvestre G, Araujo SC, Lima JB, et al. Undesirable consequences of insecticide resistance following Aedes aegypti control activities due to a dengue outbreak. PloS one. 2014;9 3:e92424.

5. Moyes CL, Vontas J, Martins AJ, Ng LC, Koou SY, Dusfour I, et al. Contemporary status of insecticide resistance in the major Aedes vectors of arboviruses infecting humans. PLoS Negl Trop Dis. 2017;11 7:e0005625.

6. Braga IA, Valle D. Aedes aegypti: vigilância, monitoramento da resistência e alternativas de controle no Brasil. Epidemiol Serv Saúde. 2007;16:295-302.

7. Valle D, Bellinato DF, Viana-Medeiros PF, Lima JBP, Martins Junior AJ. Resistance to temephos and deltamethrin in Aedes aegypti from Brazil between 1985 and 2017. Mem Inst Oswaldo Cruz. 2019;114:e180544.

8. Linss JG, Brito LP, Garcia GA, Araki AS, Bruno RV, Lima JB, et al. Distribution and dissemination of the Val1016lle and Phe1534Cys Kdr mutations in Aedes aegypti Brazilian natural populations. Parasit Vectors. 2014;7 1:25.

9. Martins AJ, Brito LP, Linss JG, Rivas GB, Machado R, Bruno RV, et al. Evidence for gene duplication in the voltage-gated sodium channel gene of Aedes aegypti. Evolution, medicine, and public health. 2013;2013 1:148-60.

10. Haddi K, Tome HVV, Du Y, Valbon WR, Nomura Y, Martins GF, et al. Detection of a new pyrethroid resistance mutation (V410L) in the sodium channel of Aedes aegypti. a potential challenge for 
mosquito control. Scientific reports. 2017;7:46549.

11. IBGE: IBGE Cidades. https://cidades.ibge.gov.br/brasil/rr/panorama (2019). 2019.

12. Lima JB, Da-Cunha MP, Da Silva RC, Galardo AK, Soares Sda S, Braga IA, et al. Resistance of Aedes aegypti to organophosphates in several municipalities in the State of Rio de Janeiro and Espirito Santo, Brazil. Am J Trop Med Hyg. 2003;68 3:329-33.

13. WHO. Monitoring and managing insecticide resistance in Aedes mosquito populations - Interim guidance for entomologists. vol. WHO/ZIKV/VC/16.1. Geneva: World Health Organization; 2016.

14. Brito LP, Carrara L, Freitas RMd, Lima JBP, et al. Levels of Resistance to Pyrethroid among Distinct kdr Alleles in Aedes aegypti Laboratory Lines and Frequency of kdr Alleles in 27 Natural Populations from Rio de Janeiro, Brazil. BioMed research international. 2018;2018:10.

15. Raymond M. Presentation d'une programme d'analyse logprobit pour microordinateur cahiers Orstrom. Sér Ent Med Parasitol. 1985;22:117-21.

16. Macoris ML, Martins AJ, Andrighetti MTM, Lima JBP, Valle D. Pyrethroid resistance persists after ten years without usage against Aedes aegypti in governmental campaigns: Lessons from Sao Paulo State, Brazil. PLoS Negl Trop Dis. 2018;12 3:e0006390.

17. Belinato TA, Martins AJ, Valle D. Fitness evaluation of two Brazilian Aedes aegypti field populations with distinct levels of resistance to the organophosphate temephos. Mem I Oswaldo Cruz. 2012;7(7):916-922.

18. Peiris HTR, Hemingway J. Temephos resistance and the associated cross-resistance spectrum in a strain of Culex quinquefasciatus Say (Diptera: Culicidae) from Peliyagoda, Sri Lanka. B Entomol Res. 1990;80 1:49-55.

19. Goindin D, Delannay C, Gelasse A, Ramdini C, Gaude T, Faucon F, et al. Levels of insecticide resistance to deltamethrin, malathion, and temephos, and associated mechanisms in Aedes aegypti mosquitoes from the Guadeloupe and Saint Martin islands (French West Indies). Infect Dis Poverty. 2017;6 1:38.

20. Viana-Medeiros PF, Bellinato DF, Valle D. Laboratory selection of Aedes aegypti field populations with the organophosphate malathion: Negative impacts on resistance to deltamethrin and to the organophosphate temephos. PLoS Negl Trop Dis. 2018;12 8:e0006734.

21. Dusfour I, Zorrilla P, Guidez A, Issaly J, Girod R, Guillaumot L, et al. Deltamethrin Resistance Mechanisms in Aedes aegypti Populations from Three French Overseas Territories Worldwide. PLoS Negl Trop Dis. 2015;9 11:e0004226.

22. Garcia GA, Sylvestre G, Aguiar R, Costa GB, Martins AJ, Lima JBP. Matching the genetics of released and local Aedes aegypti populations is critical to assure Wolbachia invasion. PLoS Negl Trop Dis. 2019;131.

23. Achee NL, Grieco JP, Vatandoost H, Seixas G, Pinto J, Ching-Ng L. Alternative strategies for mosquitoborne arbovirus control. PLoS Negl Trop Dis. 2019;131.

24. O'Neill SL, Ryan PA, Turley AP, Wilson G, Retzki K, Iturbe-Ormaetxe I, et al. Scaled deployment of Wolbachia to protect the community from dengue and other Aedes transmitted arboviruses [version 
3; peer review: 2 approved]. Gates Open Res. 2019;2:36.

25. Evans BR, Kotsakiozi P, Costa-da-Silva AL, loshino RS, Garziera L, Pedrosa MC, et al. Transgenic Aedes aegypti Mosquitoes Transfer Genes into a Natural Population. Scientific reports. 2019;9 1:13047.

26. Abad-Franch F, Zamora-Perea E, Ferraz G, Padilla-Torres SD, Luz SL. Mosquito-disseminated pyriproxyfen yields high breeding-site coverage and boosts juvenile mosquito mortality at the neighborhood scale. PLoS Negl Trop Dis. 2015;9 4:e0003702.

\section{Figures}
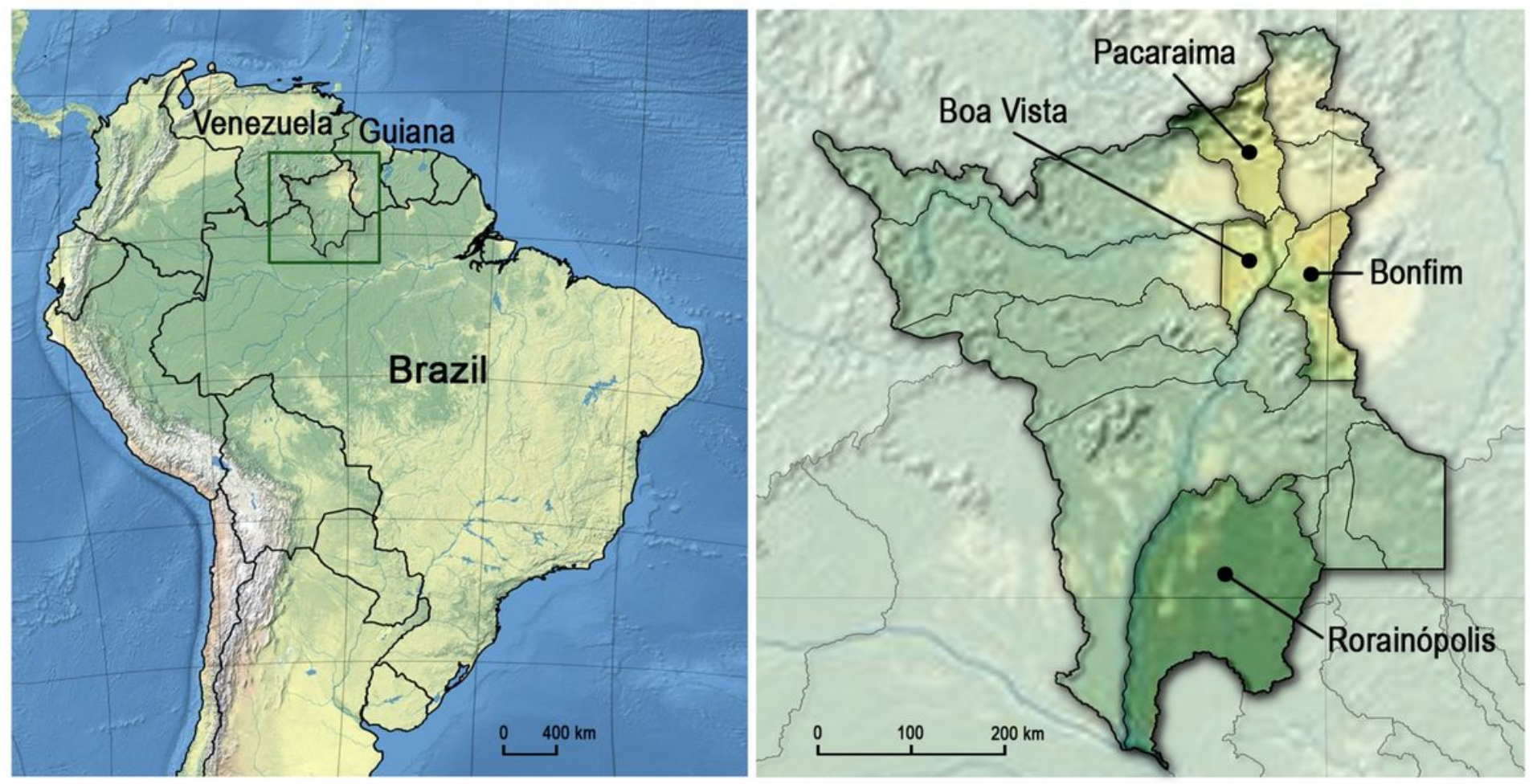

Figure 1

Localization of Roraima, the northernmost Brazilian State, evidencing its borders with Venezuela and Guyana. Boa Vista, Bonfim, Pacaraima and Rorainópolis are indicated in the magnified square. 


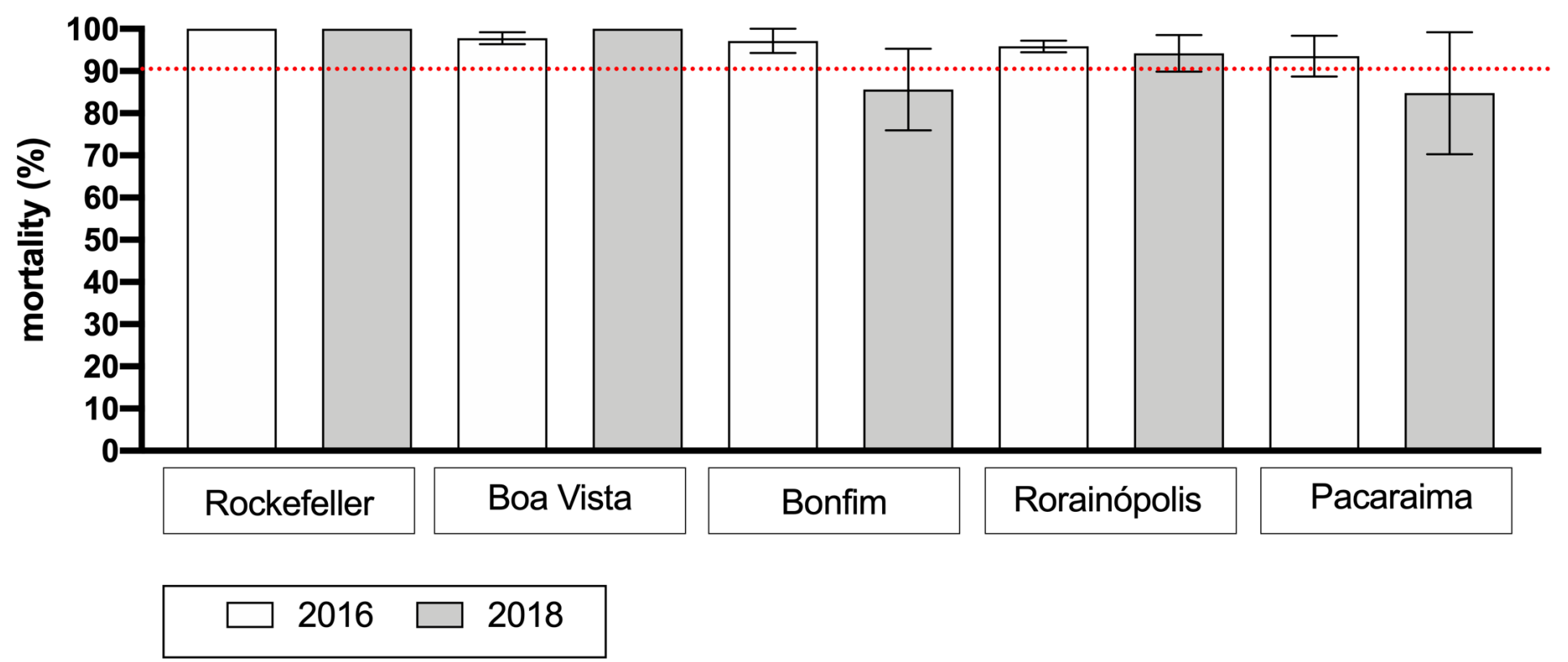

Figure 2

Evaluation of Ae. aegypti from Roraima to the organophosphate malathion. Bars indicate the mean percentage of mortality (with standard error of mean), $24 \mathrm{~h}$ post $1 \mathrm{~h}$ of exposition to malathion $0.7 \%$. Populations with mortality beyond $90 \%$ (red dotted line) are classified as resistant to the insecticide. 

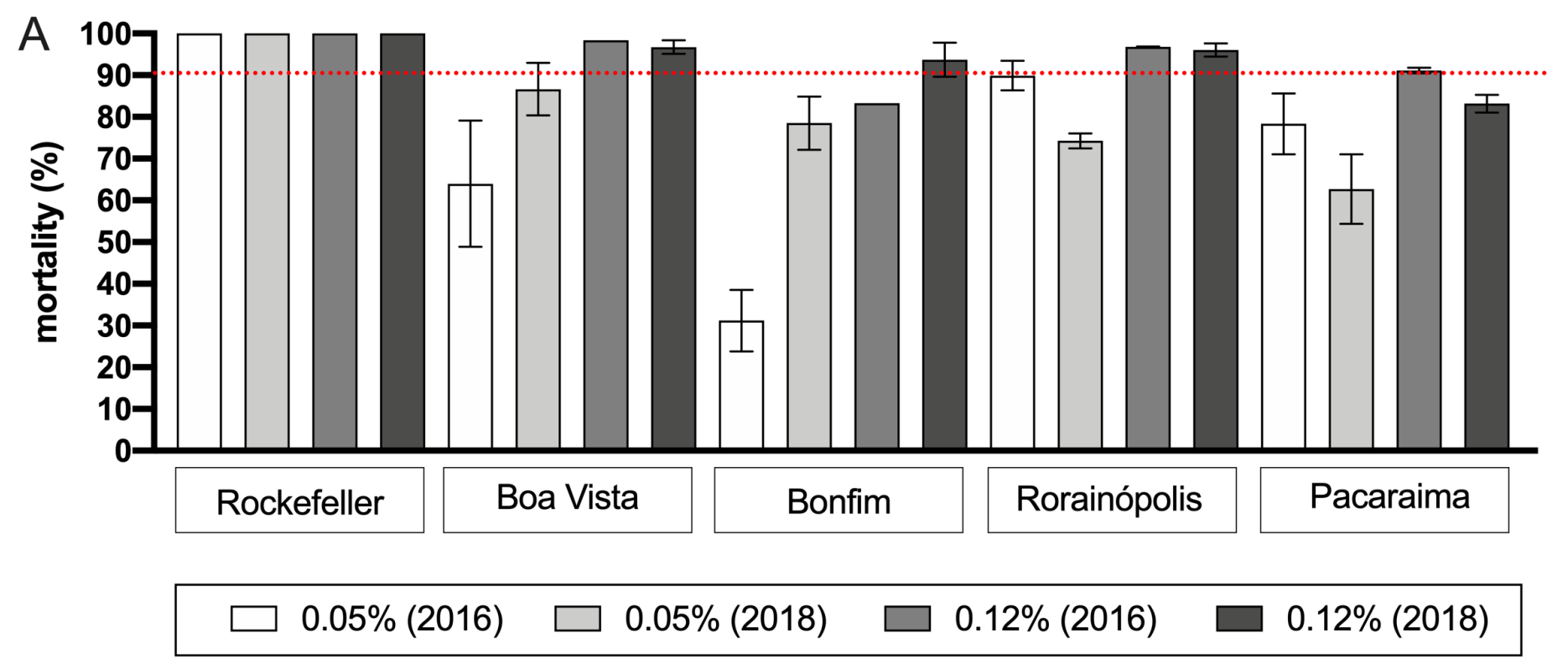

B

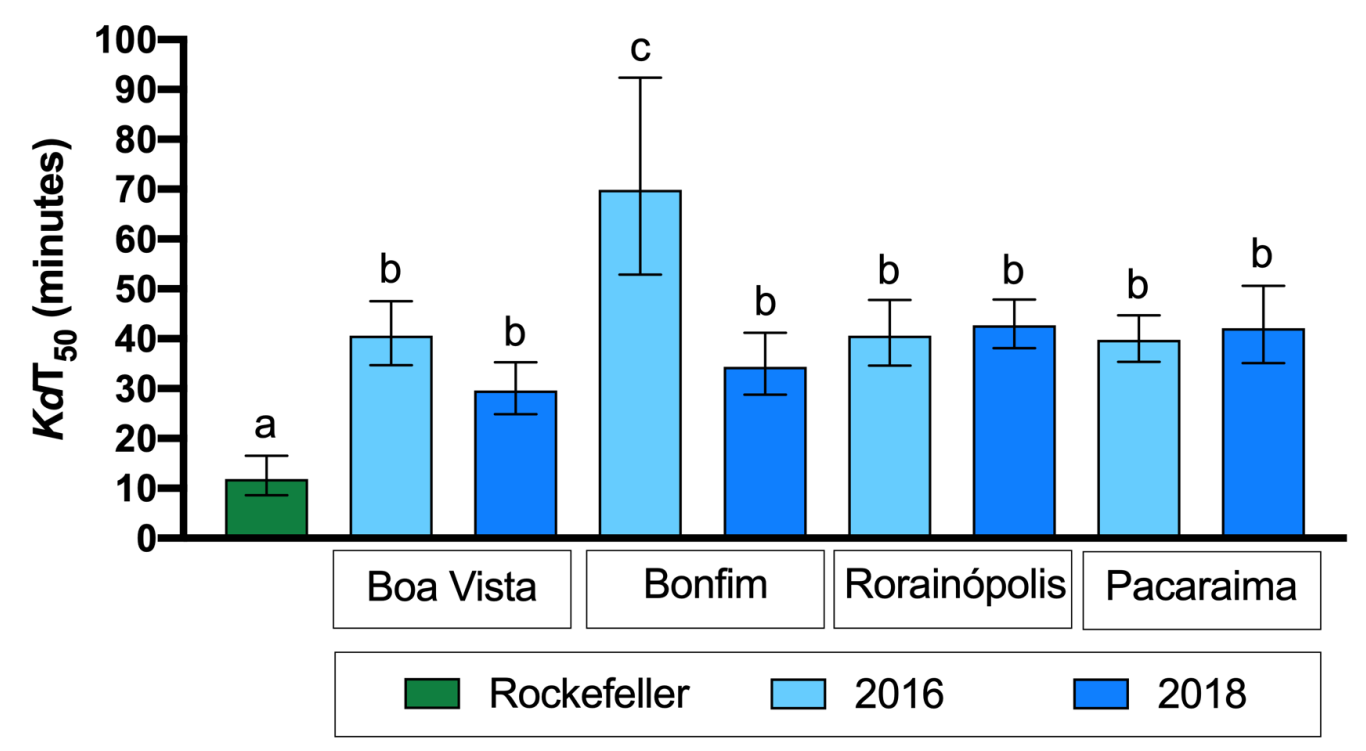

Figure 3

Evaluation of Ae. aegypti from Roraima to the pyrethroid deltamethrin. A- Bars indicate the mean percentage of mortality (with standard error of mean), 24h post $1 \mathrm{~h}$ of exposition to deltamethrin Populations with mortality beyond $90 \%$ (red dotted line) are classified as resistant to the insecticide. B KdT50 with IC95. Similar letters indicate similar times (overlapped IC95 intervals). 

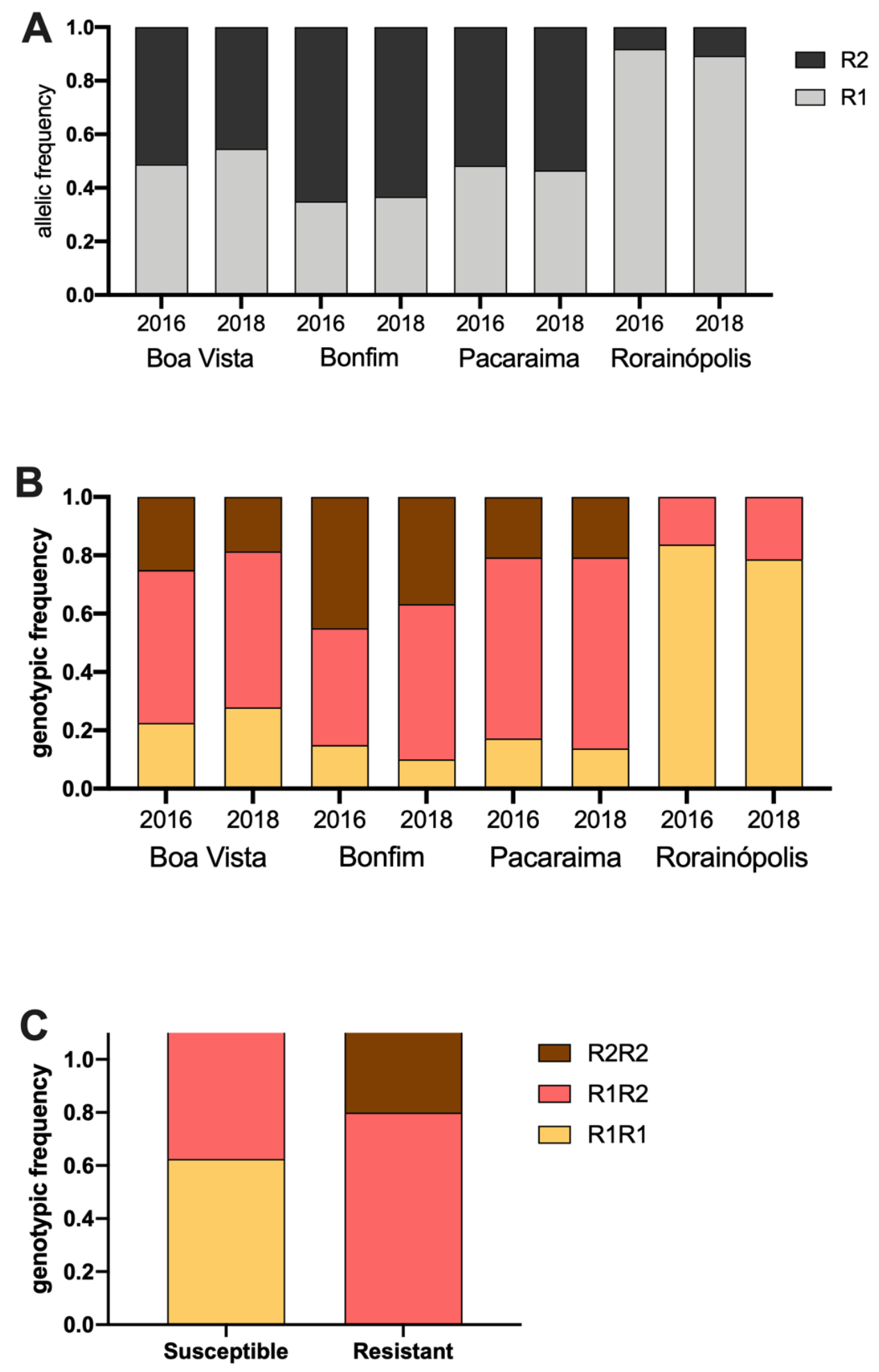

Figure 4

Genotyping of kdr mutations in Ae. aegypti populations from Roraima. A-Allelic frequencies of the kdr haplotypes. B - Genotypic frequencies. C - Genotypic frequencies of Ae. aegypti from Boa Vista and Bonfim (2016) Susceptible Resistant to deltamethrin 0.05\%.

\section{Supplementary Files}


This is a list of supplementary files associated with this preprint. Click to download.

- Graphicabstract.tiff

- Additionalfile1.pdf

- Additionalfile2.pdf 